\title{
Inhibitory effects of miR-25 targeting HMGB1 on macrophage secretion of inflammatory cytokines in sepsis
}

\author{
CHUNYAN ZHU ${ }^{1}$, TING CHEN ${ }^{2}$ and BAO LIU $^{1}$ \\ ${ }^{1}$ Intensive Care Unit, Anhui Provincial Hospital Affiliated to Anhui Medical University; \\ ${ }^{2}$ Intensive Care Unit, The Second People's Hospital of Hefei, Hefei, Anhui 230032, P.R. China
}

Received September 9, 2017; Accepted February 23, 2018

DOI: $10.3892 / \mathrm{ol} .2018 .9308$

\begin{abstract}
High mobility group box 1 (HMGB1) can promote the migration of macrophages and the release of inflammatory cytokines, functions associated with the occurrence of sepsis. The role of microRNA (miR)-25 in the targeted regulation of HMGB1 expression and the release of macrophage inflammatory cytokines remains uncharacterized. The present study investigated the association between miR-25, HMGB1 and sepsis by analyzing the expression of miR-25 and HMGB1 in patients with sepsis. The present study also investigated whether miR-25 serves a role in targeting the regulation of HMGB1 expression and macrophage inflammatory factor release. Patients with sepsis were selected from the Intensive Care Unit, and serum levels of HMGB1. The expression of miR-25 and HMGB1 in serum and peripheral blood mononuclear cells (PBMCs) was compared. Macrophages were cultured in vitro and divided into 5 groups following treatment with lipopolysaccharide (LPS). The expression levels of miR-25, HMGB1, phosphorylated (p-)p65, tumor necrosis factor- $\alpha$ (TNF- $\alpha$ ), interleukin-6 (IL-6) and HMGB-1 were compared, and the migration ability of cells was investigated by Transwell assays. Compared with the healthy controls, patients with sepsis exhibited elevated expression of HMGB1 and decreased expression of miR-25 in serum and PBMCs. Following treatment with LPS, the expression of HMGB1 and p-p65 was elevated, and the expression of miR-25 was decreased in macrophages compared with untreated cells. Following transfection with miR-25 mimics and/or short interfering RNA-HMGB1, the expression of HMGB1 in macrophages decreased significantly, the expression of p-p65, HMGB-1, TNF- $\alpha$ and IL-6 in the culture solution were also decreased, and the migration ability of macrophages was attenuated. The present study suggests that miR-25 attenuated
\end{abstract}

Correspondence to: Dr Bao Liu, Intensive Care Unit, Anhui Provincial Hospital Affiliated to Anhui Medical University, Hefei, Anhui 230032, P.R. China

E-mail: baoliu0509@126.com

Key words: microRNA-25, high mobility group box 1, nuclear factor- $\kappa \mathrm{B}$, macrophages, sepsis the induction of HMGB1 by LPS, decreased the activity of nuclear factor- $\kappa \mathrm{B}$ and the transcriptional activation of TNF- $\alpha$ and IL-6, and suppressed the migration of macrophages. Inhibiting expression of miR-25 may serve a role in upregulating HMGB1 expression, promoting the secretion of inflammatory cytokines and resulting in sepsis.

\section{Introduction}

Sepsis is a systemic inflammatory response syndrome (SIRS) caused by a variety of pathogenic microorganisms and endotoxin release; it is also a serious complication for severe patients with acute and severe trauma and shock (1). HMGB1 serves a key role in the occurrence and progression of sepsis, and its production is induced by secretions of immune cells, including mononuclear cells, dendritic cells, macrophages stimulated by endotoxins, and inflammatory cytokines (2). It is one of the most important inflammatory mediators for the lethal effect of sepsis (3). The interaction of HMGB1 with other inflammatory mediators serves an important role in mediating the signaling pathways of the inflammatory response (4). A previous study demonstrated that HMGB1 could promote the migration of macrophages and the release of various inflammatory cytokines, causing aggregation of a variety of immune cells and inducing the inflammatory responses of sepsis (3). Another study also demonstrated that the elevated expression of HMGB1 is closely associated with the occurrence of sepsis (5). miRs are highly conserved, endogenous, non-coding small RNAs, which can regulate the expression of target genes by complete or incomplete complementary pairing with the 3'-untranslated region (3'-UTR) of the mRNA, serving an important role in immune cell activation, inflammatory cytokine release and the immune response $(6,7)$. Previous research has revealed that miR-25 was involved in the occurrence and progression of sepsis and has potential as a diagnostic marker of sepsis $(8,9)$. miR-25 expression in peripheral blood in patients with sepsis is abnormal, suggesting that miR-25 abnormalities may be associated with the pathogenesis of sepsis $(1,2)$. Bioinformatics analysis revealed that a target binding site existed between miR-25 and the HMGB1 3'-UTR. However, the specific role of miR-25 in the regulation of HMGB1 and sepsis remains unclear. In the present study, by analyzing the expression of miR-25 and HMGB1 in patients with sepsis, the association between miR-25, HMGB1 and sepsis was investigated. 


\section{Materials and methods}

Reagents and materials. The Ficoll-Paque Plus was purchased from GE Healthcare (Chicago, IL, USA; cat. no. 17-1440-02). $\alpha$-MEM was from Hyclone (GE Healthcare) and fetal bovine serum was from Gibco (Thermo Fisher Scientific, Inc., Waltham, MA, USA; cat. no. 10099141). Streptomycin was from Lonza Group, Ltd. (Basel, Switzerland; cat. no. 17-602E). Lipopolysaccharide (LPS) was from Sigma-Aldrich (Merck KGaA, Darmstadt, Germany; cat. no. L6529). TRIzol (cat. no. 15596018), and Lipofectamine ${ }^{\circledR} 2000$ (cat.no. 11668019) were from Thermo Fisher Scientific, Inc. The PrimeScript $^{\mathrm{TM}} \mathrm{RT}$ reagent kit was from Takara Biotechnology Co., Ltd. (Dalian, China; cat. no. RR037A) and the SYBR Green Real-Time PCR Master mix was from Thermo Fisher Scientific, Inc., (cat. no. 4309155). miR-25 nucleotide fragments and primers were synthesized by Guangzhou RiboBio Co.,Ltd., Guangzhou, China. The mouse anti-human HMGB-1 antibody was purchased from Cell Signaling Technology, Europe, B.V. (Leiden, The Netherlands; cat. no. 6893). Mouse anti-p-NF-kB p65 was purchased from Santa Cruz Biotechnology, Inc. (Dallas, TX, USA; cat. no. sc-166748). Rabbit anti-GAPDH (cat. no. ab181602) and Lamin B1 (cat. no. ab133741) were purchased from Abcam (Cambridge, UK). Horseradish peroxidase-labeled anti-mouse (cat. no. 115-035-206) and anti-rabbit (cat. no. 323-005-021) secondary antibodies were purchased from Jackson ImmunoResearch Laboratories, Inc. (West Grove, PA, USA). Transwell chambers were purchased from EMD Millipore (Billerica, MA, USA; cat. no. PSET010R1). The pLUC Luciferase vector was purchased from Ambion; Thermo Fisher Scientific, Inc. The Dual-Luciferase ${ }^{\circledR}$ Reporter Assay system was purchased from Promega Corporation (Madison, WI, USA; cat. no. E1910). The tumor necrosis factor $\alpha$ (TNF- $\alpha$; cat. no. ELH-TNFa-1), interleukin 6 (IL-6; cat. no. ELH-IL6-1) and HMGB-1 (cat. no. ELH-HMGB1-1) ELISA detection kits were purchased from RayBiotech (Norcross, GA USA). Human recombinant M-CSF was purchased from R\&D Systems, Inc. (Minneapolis, MN, USA; cat. no. 216-MC-025). Mouse anti-human CD68 was purchased from BD Pharmingen (BD Biosciences, Biosciences, Franklin Lakes, NJ, USA; cat. no. 562117). The BCA protein quantification kit was purchased from Beyotime Institute of Biotechnology (Haimen, China; cat. no. P0010).

Clinical data. A total of 39 patients with sepsis were admitted to the ICU of our hospital between July 2015 and April 2016. All patients met the American College of Chest Physicians/Society of Critical Care Medicine diagnostic criteria for sepsis (1992) (10). These criteria include definite or suspected infection, and (i) Body temperature $>38^{\circ} \mathrm{C}$ or $<36^{\circ} \mathrm{C}$; (ii) Heart rate $>90$ beats/min; (iii) Breathing $>20$ times $/ \mathrm{min}$ or $\mathrm{pCO}_{2}<32 \mathrm{mmHg}$; (iii) White blood cell count $>12 \times 10^{9} / 1$ or $<4 \times 10^{9} / 1$, or a proportion of immature rod-shaped cells $>10 \%$. Among the 39 cases of sepsis, 22 were male and 17 were female, aged 33-65 years (mean age $43.9 \pm 12.6$ years). A total of 32 healthy subjects without signs of infection included 19 males and 13 females, aged 35-64 years (mean age, $44.6 \pm 11.8$ years). There was no significant difference in age or sex ratio between the healthy and septic patients. All specimens were collected with the informed consent of all participants. The present study was reviewed and approved by Hefei Second People's Hospital Biomedical Ethics Committee (Hefei, China).

A total of $4 \mathrm{ml}$ peripheral venous blood was collected from patients with sepsis after admission, or from healthy subjects in a fasting state, of which $2 \mathrm{ml}$ was used to isolate peripheral blood mononuclear cells (PBMCs), and $2 \mathrm{ml}$ was used to isolate serum. For serum isolation, blood was kept at room temperature for $1 \mathrm{~h}$, then centrifuged for $10 \mathrm{~min}$ at $800 \times \mathrm{g}\left(21^{\circ} \mathrm{C}\right)$. The separated upper layer was then collected and stored at $-80^{\circ} \mathrm{C}$ until further use.

Isolation of PBMCs. A total of $2 \mathrm{ml}$ blood was diluted in $2 \mathrm{ml}$ PBS, and slowly dripped onto the surface of $5 \mathrm{ml}$ Ficoll-Paque Plus. The mixture was centrifuged for $30 \mathrm{~min}$ at $300 \mathrm{x}$ g at room temperature, then the cells in the upper white layer were transferred into another $15 \mathrm{ml}$ centrifuge tube. PBS was added at a volume of 3:1 (PBS: cell solution) and mixed gently. The mixture was centrifuged for $5 \mathrm{~min}$ at $200 \mathrm{xg}$, the supernatant was discarded, and the cell pellet was resuspended in $1 \mathrm{ml}$ ice-cold 1X PBS to obtain a PMBC cell precipitate.

Induced differentiation of macrophages. PBMCs were placed in $\alpha$-MEM medium containing $10 \%$ FBS and $1 \%$ streptomycin and cultured at $37^{\circ} \mathrm{C}$ in $5 \% \mathrm{CO}_{2}$. After $24 \mathrm{~h}$, the culture supernatant was collected and non-adherent cells were removed. Medium containing $30 \mathrm{ng} / \mathrm{ml} \mathrm{M-CSF}, 10 \%$ FBS and $1 \%$ streptomycin was replenished every $72 \mathrm{~h}$ for 1 week prior to detection of the macrophage marker, cluster of differentiation 68 (CD68).

Flow cytometry analysis. Samples were prepared using a PrimeScript ${ }^{\mathrm{TM}}$ RT reagent kit according to the manufactures' instructions, (Takara Biotechnology Co., Ltd). The phenotype of macrophages was analyzed using an intracellular CD68 assay (cat. no. Y1/82A; BD Biosciences). Cells were fixed with $4 \%$ paraformaldehyde (room temperature, $20 \mathrm{~min}$ ), permeabilized by FACS permeabilizing solution (BD PharMingen, San Diego, CA) and then stained with the aforementioned reagents for $30 \mathrm{~min}$ at room temperature. Flow cytometry analysis was performed using a FACS Aria II flow cytometer (BD Biosciences). Data were analyzed with FlowJo software (version 7.6.1; FlowJo LLC, Ashland, OR, USA).

Construction of luciferase reporter gene vector and dual-luciferase reporter gene assay. The 293 cell genome was used as a template to amplify the HMGB1 3'-UTR full length fragment, or mutation-containing fragments, then cloned into the pLUC vector to transform DH5 $\alpha$ competent cells (Thermo Fisher Scientific, Inc.). The efficient plasmids were sequenced, screened and named pLUC-HMGB1-3'-UTR-wt and pLUC-HMGB1-3'-UTR-mut. The pLUC-HMGB1-3'-UTR-wt or pLUC-HMGB1-3'-UTR-mut and miR-25 mimics were co-transfected into 293 cells (Thermo Fisher Scientific, Inc.) using Lipofectamine ${ }^{\circledR} 2000$, according to the manufacturer's instructions. Luciferase activity was detected after $48 \mathrm{~h}$. Firefly and Renilla luciferase activities were measured for each sample using the Dual-Luciferase ${ }^{\circledR}$ Reporter Assay System (cat. no. E1960; Promega Corporation). 
Macrophage transfection and LPS treatment. The microRNA. org online target gene prediction tool was used to predict the relation between miR-25 and the 3'-UTR of HMGB1 mRNA (11). Therefore, the successfully differentiated macrophages were divided into 5 groups: miR-negative control (NC) transfection group, miR-25 mimic transfection group, short interfering RNA (si)-NC transfection group, si-HMGB1 group, and miR-25 mimic+si-HMGB1 group. The siRNA sequences are as follows: si-HMGB1 sense strand, 5'-CGGCCUCUG UUUGAUUUCUTT-3', si-HMGB1 antisense strand, 5'-AGA AAUCAAACAGAGGCCGTT-3'; si-NC sense strand, 5'-UUC UCCGAACGUGUCACGUTT-3', si-NC antisense strand, 5'-ACGUGACACGUUCGGAGAATT-3'; miR-25 mimic sense strand, 5'-CAUUGCACUUGUCUCGGUCUGA-3'; miR-25 mimic antisense strand, 5'-UUCAAGUAAUCCAGGAUA GGCU-3'; control sense strand, 5'-UCACAAGUCAGGCUC UUGGGAC-3', control antisense strand, 5'-ACGGGUUAG GCUCUUGGGAGCU-3'. Lipofectamine ${ }^{\circledR} 2000$ was used to transfect the cells $\left(5 \times 10^{4}\right.$ cell/well, $50 \mathrm{nM}$ si-HMGB1 or si-NC in each well) according to the manufacturer's protocol. A total of $72 \mathrm{~h}$ after the transfection, cells were sequenced to confirm transfection and used for subsequent experiments. Cells in all transfection groups were treated with $100 \mathrm{ng} / \mathrm{ml}$ LPS for $48 \mathrm{~h}$ prior to collection of cells and culture supernatants.

Detection of gene expression by reverse transcriptionquantitative polymerase chain reaction ( $R T-q P C R)$. Total RNA was isolated using TRIzol, according to the manufacturer's instructions, following extraction with $99 \%$ chloroform for $2 \mathrm{~min}$ at room temperature, precipitation with $95 \%$ isopropanol for $10 \mathrm{~min}$ at room temperature, and washing with $75 \%$ ethanol followed by centrifuging at 7,500 x g for $5 \mathrm{~min}$ at $4{ }^{\circ} \mathrm{C}$, then hydrolysis with RNAse free $\mathrm{H}_{2} \mathrm{O}$. cDNA was obtained using the PrimeScript ${ }^{\mathrm{TM}}$ RT reagent kit, according to the manufacturer's instructions, and the resulting cDNAs were stored in a $-20^{\circ} \mathrm{C}$ refrigerator. PCR was performed using Taq DNA polymerase from the SYBR Green Real-Time PCR Master mix and the following primers: miR-25, forward, 5'-CGGCGGCATTGCACTTGGTCTC-3', reverse, 5'-GTG CAGGGTCCGAGGT-3'; U6, forward, 5'-ATTGGAACGATA CAGAGAAGATT-3', reverse, 5'-GGAACGCTTCACGAA TTTG-3'; HMGB1, forward, 5'-TATGGCAAAAGCGGA CAAGG-3', reverse, 5'-CTTCGCAACATCACCAATGGA-3'; GAPDH, forward, 5'-ACAACTTTGGTATCGTGGAAGG-3', reverse, 5'-GCCATCACGCCACAGTTTC-3'. The PCR reaction contained 5.0 $\mu 1$ 2X SYBR Green Mixture, $0.5 \mu 12.5 \mu \mathrm{m} / 1$ forward primer, $0.5 \mu$ of $2.5 \mu \mathrm{m} / 1$ reverse primer, $1 \mu \mathrm{l} \mathrm{cDNA}$ and $\mathrm{ddH}_{2} \mathrm{O}$ to make the volume up to $10 \mu \mathrm{l}$. The thermocycling conditions were as follows: 40 cycles of $95^{\circ} \mathrm{C}$ for $15 \mathrm{sec}, 60^{\circ} \mathrm{C}$ for $30 \mathrm{sec}$ and $74^{\circ} \mathrm{C}$ for $30 \mathrm{sec}$ in an ABI 7500-type fluorescence quantitative PCR system (Thermo Fisher Scientific, Inc.). Results were expressed by using the $2^{-\Delta \Delta \mathrm{Cq}}$ method (12), where the number of cycles $(\mathrm{Ct})$ at which the fluorescence signal exceeded a defined threshold for GAPDH was subtracted from $\mathrm{Ct}$ values for target genes $\left(\mathrm{Ct}_{\text {targets }}-\mathrm{Ct}_{\mathrm{GAPDH}}=\Delta \mathrm{CP}\right)$, and values were calculated as $2^{-\Delta \mathrm{CP}}$ and normalized to each other.

Western blotting. Protein was extracted using radioimmunoprecipitation assay buffer (Thermo Fisher Scientific, Inc.). The protein concentration was determined using a BCA protein quantification kit, according to the manufacturer's protocol. A total of $50 \mu \mathrm{g}$ of protein was loaded for separation by $8 \%$ SDS-PAGE, then transferred into polyvinylidene difluoride membranes by electroporation for $60 \mathrm{~min}$. The membranes were blocked with $5 \%$ skimmed milk for $60 \mathrm{~min}$ at room temperature, followed by incubation with the primary antibodies (HMGB1, phosphorylated (p-)p65, GAPDH and Lamin B1 antibodies were used at dilutions of were 1:100, 1:100, $1: 800$ and $1: 300$, respectively) overnight at $4^{\circ} \mathrm{C}$, then washed 3 times PBS-Tween (PBST) prior to incubation with the HRP-labeled anti-mouse or anti-rabbit secondary antibodies (dilution, 1:8,000 dilution) for $60 \mathrm{~min}$ at room temperature. The membranes were washed 3 times in PBST, and visualized using an enhanced chemiluminescent system (Thermo Fisher Scientific, Inc.).

Detection of inflammatory cytokine content by ELISA assay. All ELISA procedures strictly followed the protocols provided by the ELISA kit manufacturer.

Detection of macrophage migration ability by Transwell assay. Collagen IV added to the upper chamber of Transwell inserts with $8-\mu \mathrm{m}$ pore size (Corning Incorporated, Corning, NY, USA). Macrophages were plated into the upper chamber $\left(5 \times 10^{5}\right)$ and medium ( $\alpha$-MEM without FBS) containing $100 \mathrm{ng} / \mathrm{ml}$ LPS was added to the lower chamber. After $48 \mathrm{~h}$ of culture, the culture solution was discarded, and the insert was washed twice with PBS, and fixed with methanol for $30 \mathrm{~min}$ at room temperature, stained by $0.1 \%$ crystal violet for $20 \mathrm{~min}$ at room temperature. The number of migratory cells was counted under a light microscope at x400 magnification.

Statistical analysis. All statistical analysis was performed using SPSS ver. 18.0 (SPSS, Inc., Chicago, IL, USA). Data are expressed as mean \pm standard deviation. Differences between 2 groups were compared using Student's t-test. One-way analysis of variance was used to compare differences between multiple groups, followed by Bonferroni correction. Pearson correlation coefficient analysis was used to observe the relation between miR-25 level in serum and sepsis. $\mathrm{P}<0.05$ was considered to indicate a statistically significant difference.

\section{Results}

The expression of HMGBI is elevated and the expression of miR-25 is decreased significantly in patients with sepsis. ELISA assay results demonstrated that the level of HMGB1 in the serum of peripheral blood in patients with sepsis was significantly increased compared with the healthy control group (Table I). Western blotting results revealed that the expression level of HMGB1 protein the PBMCs of patients with sepsis was significantly increased compared with that of healthy controls (Fig. 1A). RT-qPCR demonstrated that the expression of miR-25 in the serum and PBMCs of patients with sepsis was significantly decreased compared with that of healthy controls (Fig. 1B). Pearson correlation coefficient analysis demonstrated that the expression of miR-25 in serum of patients with sepsis was negatively correlated that of HMGB1 $(\mathrm{r}=-0.591, \mathrm{P}=0.041)$. A significant positive correlation was identified between serum HMGB1 levels and HMGB1 protein 
Table I. HMGB1 content of serum in the two groups.

\begin{tabular}{lcc}
\hline Group & Number & HMGB1 (ng/ml) \\
\hline Healthy controls & 32 & $2.17 \pm 0.23$ \\
Patients with sepsis & 39 & $29.61 \pm 4.52^{\mathrm{a}}$ \\
\hline
\end{tabular}

${ }^{\mathrm{a}} \mathrm{P}<0.05$ vs. control. HMGB1, high mobility group box- 1 .

expression in PBMCs $(\mathrm{r}=0.537, \mathrm{P}<0.001)$, and a significant negative correlation was identified between serum miR-25 expression and HMGB1 protein content $(r=-0.622, \mathrm{P}<0.001)$, as presented in Fig. 1C and D.

miR-25 inhibits HMGB1 expression. MicroRNA.org online target gene prediction results indicated that there was a targeted binding site between miR-25 and the 3'-UTR of HMGB1 mRNA (Fig. 2A). Transfection of miR-25 mimics significantly reduced the relative luciferase activity, suggesting that miR-25 could target the 3'-UTR region of HMGB1 and inhibit its expression (Fig. 2B).

LPS induces macrophages to secrete HMGB1 and inhibit $m i R-25$ expression. The results of flow cytometry demonstrated that the macrophage-specific marker, CD68, was increased by $>80 \%$ following a 7-day induction with M-CSF, indicating that the differentiation of macrophages was induced successfully, allowing subsequent experiments to be performed (Fig. 3A). ELISA assays demonstrated that, following treatment with $100 \mathrm{ng} / \mathrm{ml}$ LPS for $48 \mathrm{~h}$, the protein levels of HMGB-1, TNF- $\alpha$ and IL-6 in the culture supernatant were significantly increased (Fig. 3B). The results of RT-qPCR demonstrated that LPS treatment significantly upregulated the expression of HMGB1 mRNA and significantly decreased the expression of miR-25 in macrophages (Fig. 3C). Western blotting results revealed that LPS treatment significantly enhanced the transcriptional activity of $\mathrm{NF}-\kappa \mathrm{B}$ and upregulated the expression of HMGB1 protein in the nucleus and cytoplasm of macrophages (Fig. 3D).

Elevated miR-25 could inhibit HMGB1 expression and migration in macrophages. The transfection of miR-25 mimics and/or si-HMGB1 significantly reduced HMGB1 mRNA expression (Fig. 4A) and attenuated protein expression in macrophages (Fig. 4B), resulting in a reduction in p-p65 protein expression (Fig. 4B). In addition, the levels of inflammatory cytokines, HMGB1, TNF- $\alpha$ and IL-6, were significantly decreased in culture supernatant (Fig. 4C), and the migration ability of macrophages was also significantly attenuated (Fig. 4D) in cells transfected with si-HMGB1 or miR-25 mimic + si-HMGB1 compared with control.

\section{Discussion}

The causes of sepsis are diverse, the disease has a complicated pathology and manifests as an acute and critical condition (13). A large number of pathogenic toxins activate the human immune system to produce cytokines and inflammatory responses, leading to irreversible and serious damage to cells, tissues and organs, and the immune system (14). If sepsis is not treated in a timely and effective manner, it is likely to develop into septic shock, acute respiratory distress syndrome and multiple organ dysfunction syndrome (15). Although modern medical techniques have greatly improved, the mortality rate of patients with sepsis remains high, and is the leading cause of mortality in intensive care (16). Therefore, it is of great importance to be able to diagnose sepsis early, and make timely and effective interventions, so as to delay the progression of disease and reduce the mortality rate of patients with sepsis.

HMG (high mobility group protein) is a non-histone chromatin-associated protein family, comprising HMGB1, HMGB2 and HMGB3 (17). HMGB1 is the most abundant and highly conserved HMG protein (18). It functions in a variety of biological processes, including DNA replication, DNA damage repair, transcription and translation (19). It has been revealed that HMGB1 is an important inflammatory cytokine, with an important role in mediation of inflammatory responses (20). A number of studies have demonstrated that HMGB1 serves a critical role in the initiation of an inflammatory signaling cascade in sepsis, and that it is the most important pro-inflammatory cytokine of the lethal effect of sepsis (21). Compared with inflammatory cytokines involved in early sepsis, including TNF- $\alpha$, HMGB1 is characterized by late elevated expression for a prolonged duration, providing a wide window of time for the clinical treatment of sepsis (22). As an innate immune cell, macrophages serve a crucial role in fighting against pathogenic microorganisms and initiating an immune response (23). During sepsis, macrophages synthesize and secrete HMGB1, which can promote the induction and amplification of inflammatory cytokines through positive feedback, and exacerbate and prolong the body inflammatory response process (24). As important epigenetic regulatory molecules, miRs serve an important role in immune cell proliferation, differentiation, activation, release of inflammatory cytokines and regulation of immune responses. The abnormal expression of various miRs has been associated with the occurrence of sepsis, and may be used as an important diagnostic and prognostic marker of sepsis (25). Studies have demonstrated that patients with sepsis exhibit abnormal expression of miR-25 in their peripheral blood $(1,2)$. In the present study, bioinformatics analysis revealed a target binding site between miR-25 and HMGB1 3'-UTR. It was also investigated whether miR-25 serves a role in the targeted regulation of HMGB1 expression and release of macrophage inflammatory cytokines.

In the present study, it was demonstrated that the serum HMGB1 content and the expression of HMGB1 protein in PBMCs of patients with sepsis were significantly increased compared with those of healthy controls. RT-qPCR demonstrated that the expression of miR-25 in the serum and PBMCs of patients with sepsis was significantly decreased compared with that of healthy controls. These results suggest that the decreased expression of miR-25 may be associated with the increased expression of HMGB1 and the occurrence of sepsis. Studies by Huang et al (3) also indicated that the serum level of HMGB1 in patients with sepsis was significantly elevated. Karlsson et al (26) demonstrated that increased HMGB1 of patients with sepsis was higher than that of healthy controls by over 6 times. Yao et al (25) demonstrated that expression 
B
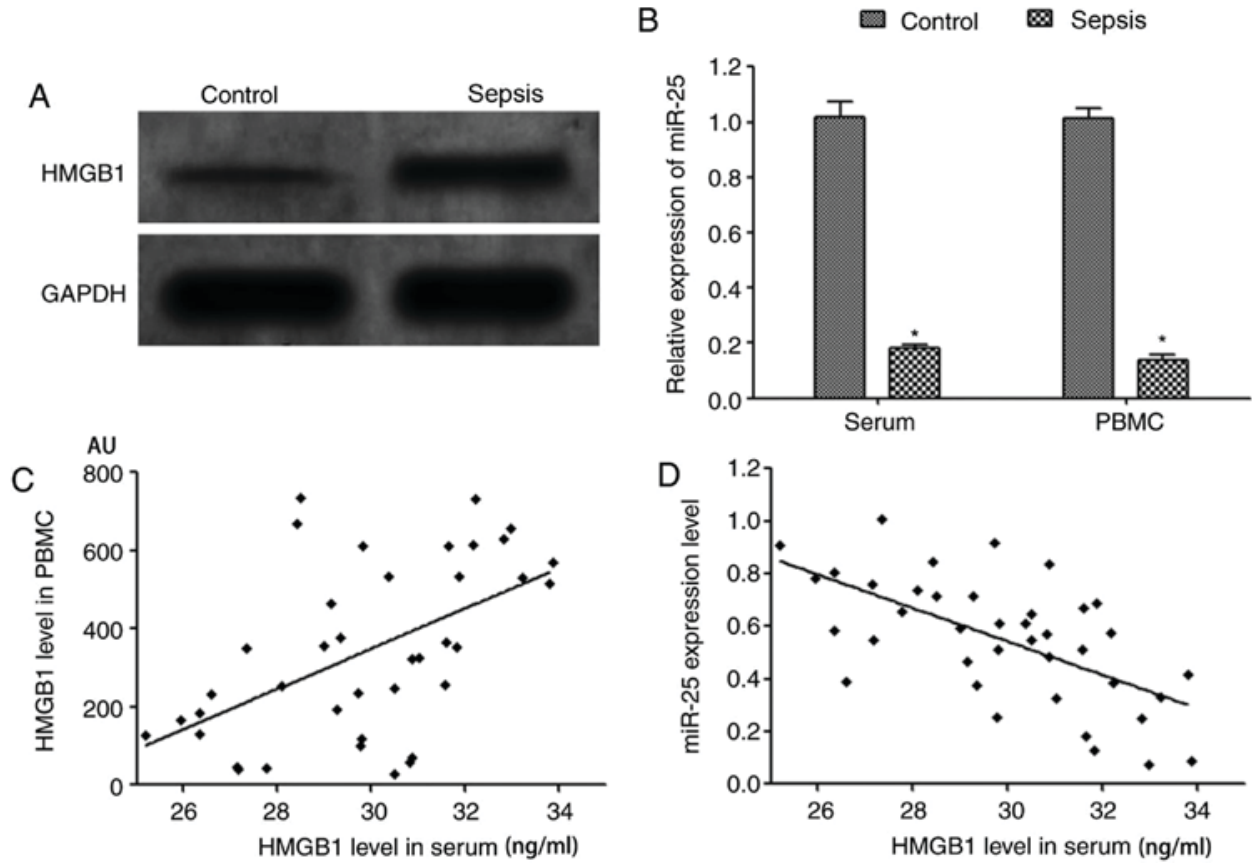

Figure 1. HMGB1 expression is increased and miR-25 expression is decreased in patients with sepsis. (A) Detection of HMGB1 protein expression by western blotting. (B) Reverse transcription-quantitative polymerase chain reaction detection of miR-25 mRNA expression. (C) The correlation between serum HMGB1 levels and HMGB1 protein expression level in peripheral blood mononuclear cells. (D) The correlation between serum miR-25 levels and HMGB1 protein expression level measured in serum. ${ }^{*} \mathrm{P}<0.05$ vs. control. HMGB1, high mobility group box-1; miR-25, microRNA-25.
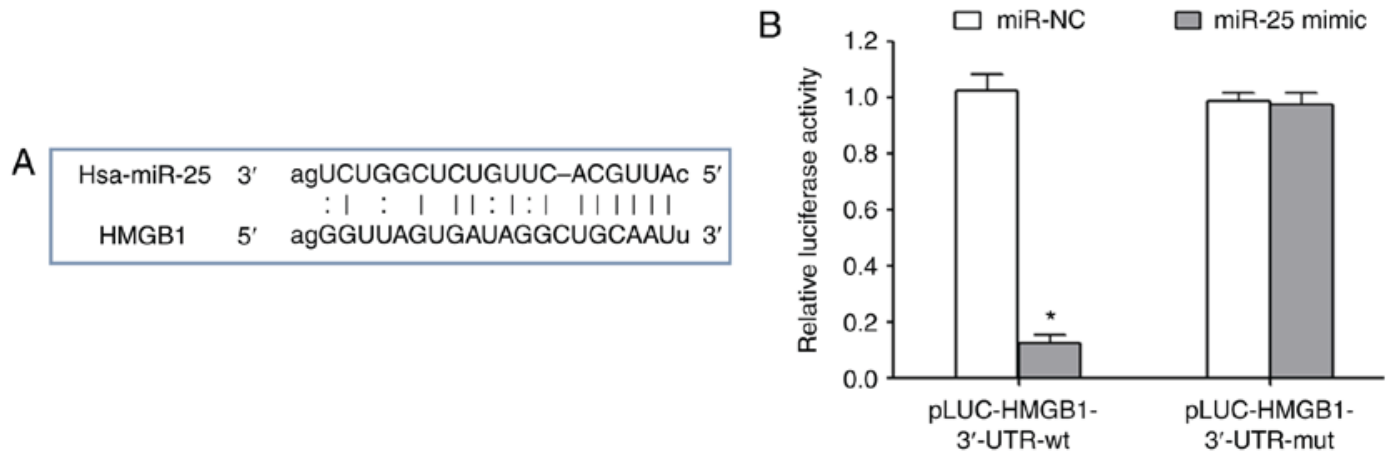

Figure 2. miR-25 inhibits HMGB1 expression. (A) The miR-25 binding site on the 3'-UTR of HMGB1 mRNA. (B) Dual luciferase reporter assay results, demonstrating the activity of the miR-25 mimics relative to miR-NC suggesting miR-25 may target the 3'-UTR region of HMGB1 and inhibit its expression. ${ }^{*} \mathrm{P}<0.05$ vs. miR-NC. miR-25, microRNA-25; HMGB1, high mobility group box 1; 3'-UTR, 3' untranslated region; NC, negative control; wt, wild type; mut, mutated.

of miR-25 in the peripheral blood of patients with sepsis was significantly decreased compared with healthy controls, and that the lower the expression of miR-25, the lower the 28-day survival rate of patients. Dual luciferase reporter assays demonstrated that transfection of miR-25 mimics significantly reduced the relative luciferase activity in 293 cells, indicating that miR-25 could target the 3 '-UTR region of HMGB1 and inhibit its expression.

The NF- $\kappa B$ signal transduction serves a key role in the initiation of the immune response and the release of inflammatory cytokines, as well as the occurrence of sepsis. Toll-like receptors (TLRs) are an important family of receptors of HMGB1. Toll-like receptors such as TLR2 and 4, which are expressed on the surface of macrophages, interact with HMGB1 and activate the NF- $\mathrm{KB}$ signaling pathway, to promote the transcription, synthesis and secretion of inflammatory cytokines, including
TNF- $\alpha$, IL-1, IL-6, and chemokines $(27,28)$. In the present study, LPS treatment significantly upregulated the expression of HMGB1 in macrophages, enhanced the transcriptional activity of NF- $\kappa \mathrm{B}$ and the release of TNF- $\alpha$ and IL-6, and inhibited the expression of miR-25. Zhang (24) indicated that LPS treatment significantly induced the synthesis and secretion of HMGB1 by macrophages. Furthermore, Zhou et al (8) demonstrated that LPS treatment significantly increased the HMGB1 expression in macrophages. In consistence with these results, the present study also demonstrated that LPS treatment significantly upregulated the expression of HMGB1 in macrophages. Transfection with miR-25 mimics and/or si-HMGB1 significantly decreased the expression of HMGB1 in macrophages, reduced the transcriptional activity of the NF- $\mathrm{KB}$ signaling pathway, and reduced the HMGB1, TNF- $\alpha$ and IL-6 levels in the culture supernatant. Decreased expression 

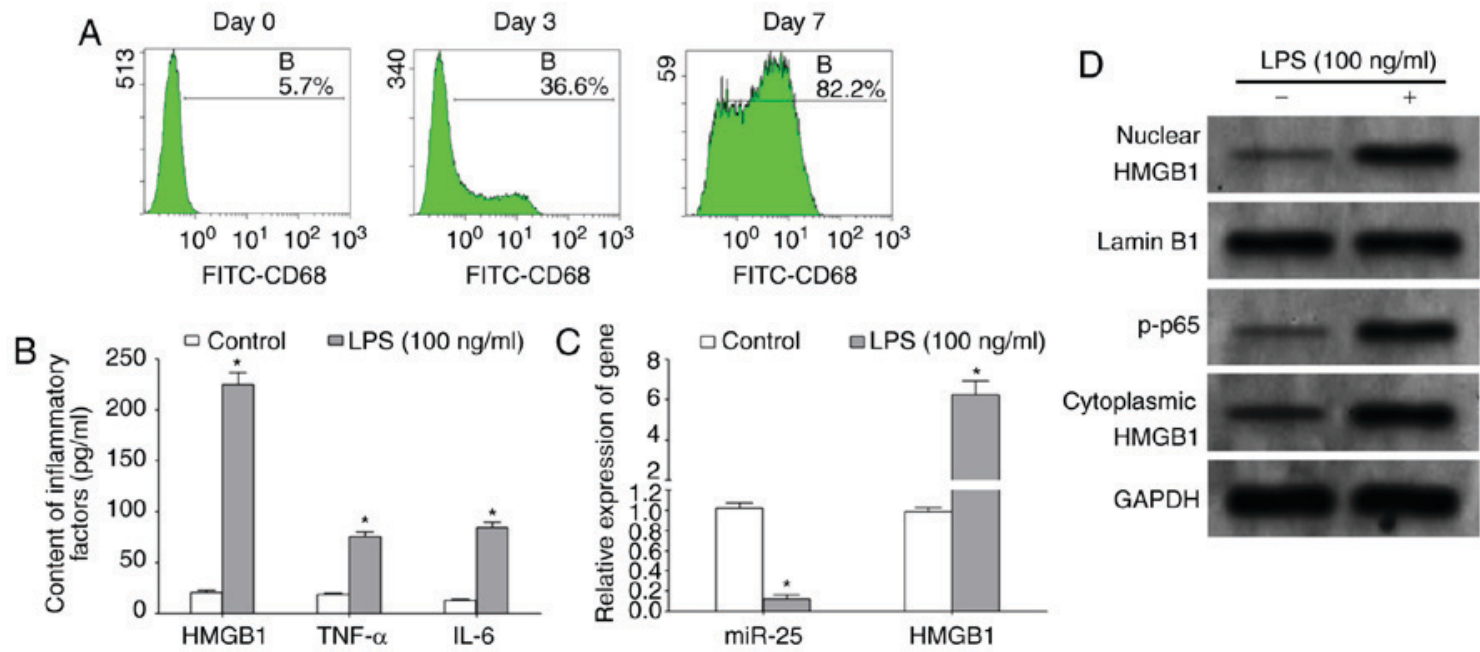

Figure 3. LPS induces macrophages to secrete HMGB1 and inhibit miR-25 expression. (A) Detection of macrophage markers CD68 by flow cytometry. (B) Test for inflammatory cytokine content by ELISA. (C) Reverse transcription-quantitative polymerase chain reaction detection of miR-25 and HMGB1 gene expression. (D) Western blotting to indicate the effect of LPS treatment on protein expression. " $\mathrm{P}<0.05$ vs. control. LPS, lipopolysaccharide; HMGB1, high mobility group box 1; miR-25, microRNA-25; CD68, cluster of differentiation 68; TNF- $\alpha$, tumor necrosis factor $\alpha$; IL-6, interleukin 6; p-, phosphorylated.
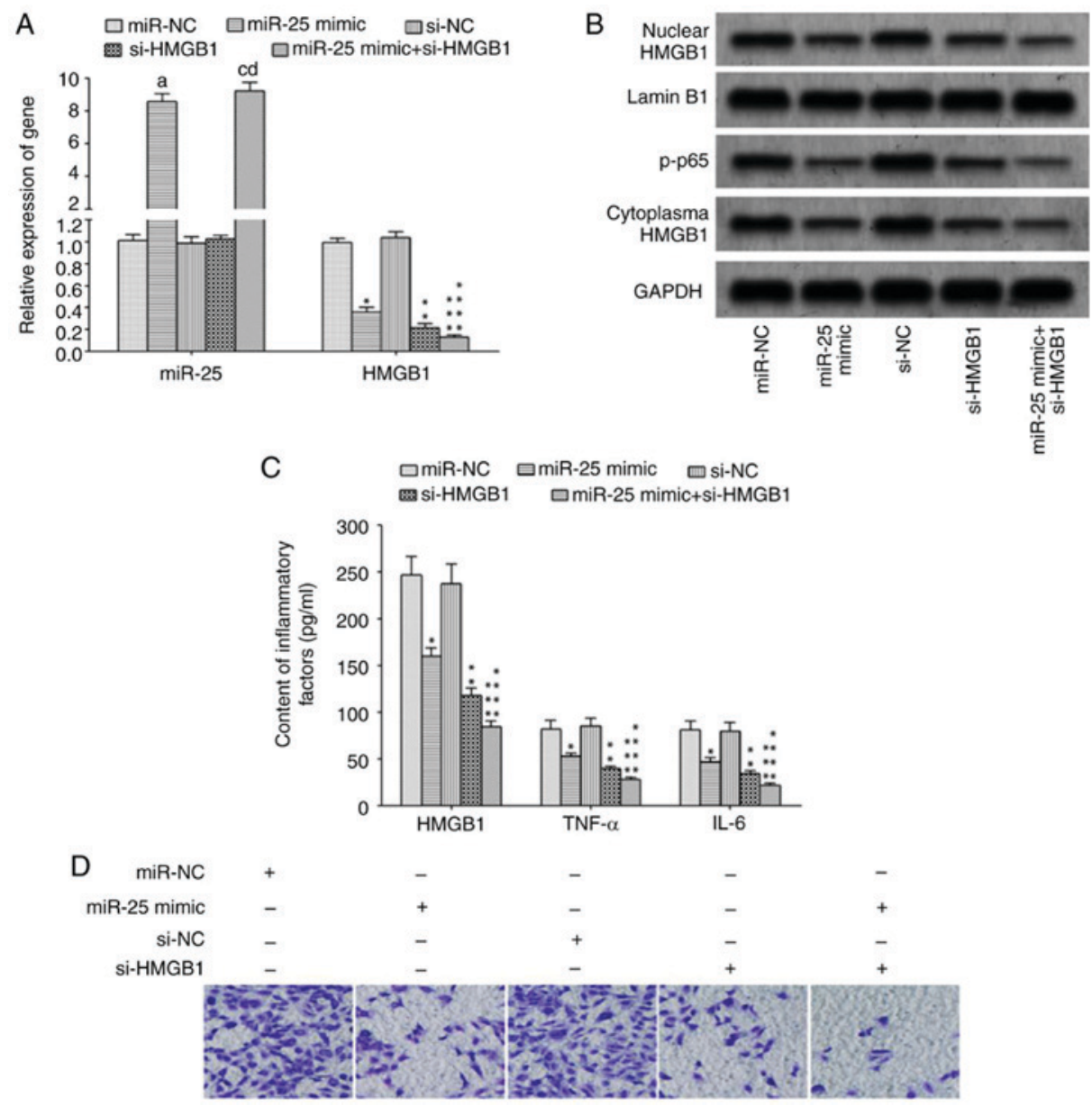

Figure 4. Elevated miR-25 expression inhibits HMGB1 expression and migration in macrophages. (A) Reverse transcription-quantitative polymerase chain reaction detection of miR-25 and HMGB1 gene expression. (B) Western blotting detection of HMGB1 and p-p65 protein expression. (C) Detection of inflammatory cytokine content by ELISA. (D) Detection of macrophage migration by Transwell assay. " miR-25 mimic vs. miR-NC, P<0.05; "*si-HMGB1 vs. si-NC, $\mathrm{P}<0.05 ;{ }^{* * *}$ miR-25 mimic + si-HMGB1 vs. miR-NC, P<0.05; *****miR-25 mimic + si-HMGB1 vs. si-NC, P<0.05. miR-25, microRNA-25; HMGB1, high mobility group box 1; p-, phosphorylated; NC, negative control; si, short interfering RNA.

of miR-25 was associated with the abnormal expression of HMGB1 following LPS treatment. Elevated expression of
miR-25 weakened the induction effect of LPS on HMGB1, and attenuated the transcriptional activity of NF- $\mathrm{KB}$ and 
the transcriptional activation of downstream inflammatory cytokines, TNF- $\alpha$ and IL-6. Transfection with miR-25 mimics and/or si-HMGB1 also attenuated the migratory ability of macrophages, which may be associated with decreased activation of the NF- $\kappa \mathrm{B}$ signaling pathway following reduction of HMGB1 expression. Furthermore, HMGB1 may bind $\mathrm{C}-\mathrm{X}-\mathrm{C}$ motif chemokine ligand 12 and activate chemokine receptor $\mathrm{C}-\mathrm{X}-\mathrm{C}$ motif chemokine receptor 4 to induce the proliferation and migration of immune cells, thus amplifying the inflammatory response. However, this hypothesis requires further investigation.

To conclude, the present study demonstrated that miR-25 attenuated the induction of HMGB1 via LPS, decreased the transcriptional activity of $\mathrm{NF}-\kappa \mathrm{B}$ and the transcriptional activation of downstream inflammatory cytokines, TNF- $\alpha$ and IL-6, and also suppressed macrophage migration.

\section{References}

1. Caserta S, Kern F, Cohen J, Drage S, Newbury SF and Llewelyn MJ: Circulating plasma microRNAs can differentiate human sepsis and systemic inflammatory response syndrome (SIRS). Sci Rep 6: 28006, 2016.

2. Wang H, Ward MF and Sama AE: Targeting HMGB1 in the treatment of sepsis. Expert Opin Ther Targets 18: 257-268, 2014

3. Huang W, Tang Y and Li L: HMGB1, a potent proinflammatory cytokine in sepsis. Cytokine 51: 119-126, 2010.

4. Huston JM, Wang H, Ochani M, Ochani K, Rosas-Ballina M, Gallowitsch-Puerta M, Ashok M, Yang L, Tracey KJ and Yang H: Splenectomy protects against sepsis lethality and reduces serum HMGB1 levels. J Immunol 181: 3535-3539, 2008.

5. Wang SY, Li ZJ, Wang X, Li WF and Lin ZF: Effect of ulinastatin on HMGB1 expression in rats with acute lung injury induced by sepsis. Genet Mol Res 14: 4344-4353, 2015.

6. Bulun SE and Nezhat C: Aromatase, microRNA, and inflammation: A complex relationship. Fertil Steril 106: 552-553, 2016.

7. Wang X, Guo Y, Wang C, Yu X and Yu H: MicroRNA-142-3p inhibits chondrocyte apoptosis and inflammation in osteoarthritis by targeting HMGB1. Inflammation 39: 1718-1728, 2016.

8. Zhou W, Wang J, Li Z, Li J and Sang M: MicroRNA-2055b inhibits HMGB1 expression in LPS-induced sepsis. Int J Mol Med 38: 312-318, 2016.

9. Benz F, Roy S, Trautwein C, Roderburg C and Luedde T: Circulating microRNAs as biomarkers for sepsis. Int $\mathrm{J}$ Mol Sci 17: 78, 2016.

10. Weiss M, Huber-Lang M, Taenzer M, Traeger K, Altherr J, Kron M, Hay B and Schneider M: Different patient case mix by applying the $2003 \mathrm{SCCM} / \mathrm{ESICM} / \mathrm{ACCP} / \mathrm{ATS} / \mathrm{SIS}$ sepsis definitions instead of the 1992 ACCP/SCCM sepsis definitions in surgical patients: A retrospective observational study. BMC Med Inform Decis Mak 9: 25, 2009.

11. Ritchie W, Rasko JE and Flamant S: MicroRNA target prediction and validation. Adv Exp Med Biol 774: 39-53, 2013.

12. Livak KJ and Schmittgen TD: Analysis of relative gene expression data using real-time quantitative PCR and the 2- $\Delta \Delta C \mathrm{~T}$ method. Methods 25: 402-408, 2001.

13. Boyd JH, Russell JA and Fjell CD: The meta-genome of sepsis: Host genetics, pathogens and the acute immune response. J Innate Immun 6: 272-283, 2014.
14. Czura CJ, Yang H, Amella CA and Tracey KJ: HMGB1 in the immunology of sepsis (not septic shock) and arthritis. Adv Immunol 84: 181-200, 2004.

15. Lin SM, Chung FT, Kuo CH, Chou PC, Wang TY, Chang PJ, Lo YL, Huang CD, Lin HC, Wang CH and Kuo HP: Circulating angiopopietin-1 correlates with the clinical course of multiple organ dysfunction syndrome and mortality in patients with severe sepsis. Medicine (Baltimore) 94: e878, 2015.

16. Guirgis FW, Khadpe JD, Kuntz GM, Wears RL, Kalynych CJ and Jones AE: Persistent organ dysfunction after severe sepsis: A systematic review. J Crit Care 29: 320-326, 2014.

17. Chavan SS, Huerta PT, Robbiati S, Valdes-Ferrer SI, Ochani M, Dancho M, Frankfurt M, Volpe BT, Tracey KJ and Diamond B: HMGB1 mediates cognitive impairment in sepsis survivors. Mol Med 18: 930-937, 2012.

18. Gentile LF and Moldawer LL: HMGB1 as a therapeutic target for sepsis: It's all in the timing! Expert Opin Ther Targets 18: 243-245, 2014.

19. Shao J, Zhao M, Tong M, Wei J, Wise MR, Stone P, Chamley L and Chen Q: Increased levels of HMGB1 in trophoblastic debris may contribute to preeclampsia. Reproduction 152: 775-784, 2016.

20. Chang KC, Ko YS, Kim HJ, Nam DY and Lee DU: 13-Methylberberine reduces HMGB1 release in LPS-activated RAW264.7 cells and increases the survival of septic mice through AMPK/P38 MAPK activation. Int Immunopharmacol 40: 269-276, 2016

21. Zhao H, Liu Z, Liu W, Han X and Zhao M: Betulin attenuates lung and liver injuries in sepsis. Int Immunopharmacol 30: 50-56, 2016.

22. Gil M, Kim YK, Hong SB and Lee KJ: Naringin decreases TNF- $\alpha$ and HMGB1 release from LPS-stimulated macrophages and improves survival in a CLP-induced sepsis mice. PLoS One 11: e0164186, 2016.

23. Kumar V: Targeting macrophage immunometabolism: Dawn in the darkness of sepsis. Int Immunopharmacol 58: 173-185, 2018

24. Zhang Z, Zhang L, Zhou $\mathrm{C}$ and Wu H: Ketamine inhibits LPS-induced HGMB1 release in vitro and in vivo. Int Immunopharmacol 23: 14-26, 2014.

25. Yao L, Liu Z, Zhu J, Li B, Chai C and Tian Y: Clinical evaluation of circulating microRNA-25 level change in sepsis and its potential relationship with oxidative stress. Int J Clin Exp Pathol 8: 7675-7684, 2015

26. Karlsson S, Pettila V, Tenhunen J, Laru-Sompa R, Hynninen M and Ruokonen E: HMGB1 as a predictor of organ dysfunction and outcome in patients with severe sepsis. Intensive Care Med 34: 1046-1053, 2008.

27. Chen XL, Sun L, Guo F, Wang F, Liu S, Liang X, Wang RS, Wang YJ and Sun YX: High-mobility group box-1 induces proinflammatory cytokines production of Kupffer cells through TLRs-dependent signaling pathway after burn injury. PLoS One 7: e50668, 2012.

28. Mudaliar H, Pollock C, Komala MG, Chadban S, Wu H and Panchapakesan U: The role of toll-like receptor proteins (TLR) 2 and 4 in mediating inflammation in proximal tubules. Am J Physiol Renal Physiol 305: F143-F154, 2013.

This work is licensed under a Creative Commons Attribution-NonCommercial-NoDerivatives 4.0 International (CC BY-NC-ND 4.0) License. 